\title{
Los cabos atados y sueltos en los estudios agrarios y étnicos en Ecuador
}

\author{
Tying up Loose Ends on Agricultural and Ethnic \\ Studies in Ecuador
}

\author{
Fernando GARCÍA \\ FLACSO Ecuador \\ fgarcia@flacso.edu.ec
}

Recibido: 31 de enero de 2014

Aceptado: 4 de agosto de 2014

\section{Resumen}

En este ensayo se analiza que, a pesar del avance en el reconocimiento de los derechos colectivos de las nacionalidades y pueblos indígenas y afroecuatorianos, en Ecuador persisten procesos de desigualdad económica y de exclusión étnica y cultural. Para ello, se revisan siete temas vinculados a los estudios agrarios y étnicos ecuatorianos desarrollados en los últimos cincuenta años: el acceso a la tierra, la fuerza de trabajo campesina, las relaciones étnicas y de clase, la noción de comunidad, el racismo, la participación de las mujeres y la relación entre los organismos internacionales, Estado y movimientos sociales.

Palabras clave: Estudios Agrarios; Estudios Étnicos; Nacionalidades; Pueblos Indígenas; Afrodescendientes; Identidad; Desigualdad; Ecuador.

\begin{abstract}
In spite of advances in the recognition of the collective rights of indigenous nationalities and Afro-Ecuadorians, there are still processes of economic inequality and ethnic and cultural exclusion in Ecuador. For this reason, this paper take into account seven issues linked to agricultural and Ecuadorian ethnic studies developed in the last fifty years: access to land, rural labor force, ethnic and class relations, the notion of community, racism, women's participation and the relationship between international agencies, state and social movements.
\end{abstract}

Keywords: Agrarian Studies; Ethnic Studies; Indigenous People; Afro-Ecuadorian; Identity; Inequality; Ecuador.

Referencia normalizada: García, F. (2014). Los cabos atados y sueltos en los estudios agrarios y étnicos en Ecuador. Revista de Antropología Social, 23, pp. 73-89.

SUMARIO: 1. Introducción. 2. Los puntos de quiebre entre los estudios étnicos y agrarios en Ecuador. 3. Referencias bibliográficas. 
Identifico dos sistemas de pertenencia y subordinación en las sociedades capitalistas modernas: desigualdad y exclusión. De acuerdo al primero, las personas están subordinadas por el modo en que se integran a la sociedad mientras que, en el segundo caso, se encuentran subordinadas por el modo en que son excluidas de la sociedad. Mientras la desigualdad se establece por el principio de igualdad, la exclusión se establece de acuerdo al principio de la diferencia (Santos 2003:175)

\section{Introducción}

Este ensayo parte del hecho que para superar la desigualdad y la exclusión es necesario buscar una nueva articulación entre políticas de igualdad y políticas de identidad. En ese sentido, pretendo analizar el desarrollo de los estudios agrarios y étnicos en Ecuador utilizando un doble contrapunto que coincide con los énfasis puestos en estos estudios en los últimos cincuenta años. Por un lado, el intento del mundo campesino de lograr la tan ansiada igualdad económica a través del acceso a la propiedad de la tierra y la venta de su fuerza trabajo en el mercado interno y, por otro, el intento del mundo indígena de superar la desigualdad y la exclusión a través de la afirmación de su identidad y de la venta de su fuerza de trabajo en el mercado tanto nacional como internacional.

En el transcurso del texto mostraré que ambos campos de estudio y sus actores no solo siguen vigentes en el país, sino que muestran diferentes intersecciones que han permitido que muchos cabos sueltos se aten y otros, en cambio, continúen todavía sueltos. El enfoque que privilegiaré será el desarrollado al interior de la antropología ecuatoriana desde estos dos campos de estudio, sin dejar de lado el aporte hecho a la discusión desde las otras disciplinas de las ciencias sociales. Siguiendo el marco histórico de estos estudios en la primera parte, se hará referencia a lo sucedido entre la década de los sesenta y los noventa en el campo agrario y étnico, y lo mismo a partir de la década de los noventa hasta a la actualidad. En ambos períodos pondré especial atención a los puntos de encuentro y de desencuentro para poder obtener una visión crítica del debate.

Antes de iniciar el análisis es conveniente hacer una breve contextualización de la situación del Ecuador en términos demográficos, regionales y de composición étnica. Según el Censo de 2010, el país tenía una población de 14.483 .499 ecuatorianos y ecuatorianas distribuida en el sector urbano el 62.76\% (9.090.786) y en el sector rural el 37.24\% (5.392.713). La composición étnica de este conjunto corresponde a: 71,93\% mestizos (10.417.299), 7,39\% montubios ${ }^{1}(1.070 .728), 7,19 \%$ afroecuatorianos (1.041.599), 7,03\% indígenas (1.018.176) y otros $0.37 \%(53.354)$ (INEC, 2010). cultural.

${ }^{1}$ La Constitución ecuatoriana de 2008 reconoció al pueblo montubio como grupo étnico y 


\section{Los puntos de quiebre entre los estudios étnicos y agrarios en Ecuador}

En el inicio de la profesionalización e institucionalización de la antropología ecuatoriana a inicios de los años setenta ${ }^{2}$, una de las líneas de investigación prioritarias (Martínez, 2007) fue la vinculada con los estudios campesinos. Estos trabajos se inspiraron principalmente en las obras planteadas desde el marxismo y la antropología política de la época (A. V. Chayanov, 1974; Eric R. Wolf, 1977 y Sidney Mintz, 1973), y basaron sus temáticas en la diversidad cultural y étnica, con la especificidad que Angel Palerm y sus seguidores introdujeron en sus investigaciones desde México, es decir, la necesaria vinculación entre la antropología y la historia por un lado, y por otro, la práctica investigadora basada en largas temporadas de trabajo de campo. Así, si se revisan los títulos de las tesis de los primeros estudiantes graduados en el Departamento de Antropología de la Universidad Católica del Ecuador, el tema de la economía y la política campesina es reiterativo.

Estas elaboraciones se dieron en el marco del proceso de reforma agraria iniciado en 1964 con la primera ley, y fue cerrado treinta años más tarde con la ley de Desarrollo Agropecuario. La discusión central en la época, muy propia de los estudios marxistas y de la izquierda, era si los campesinos ecuatorianos adoptarían la vía farmer o la vía yunker en el proceso de transición de las relaciones precapitalistas de producción a otras netamente capitalistas ${ }^{3}$. Algunos autores más campesinistas planteaban que el mantenimiento de las estrategias culturales propias de los campesinos andinos (reciprocidad, parentesco y la vigencia de la comunidad), les iba a permitir mantener una especie de resistencia al avance del capital, mientras que otros, en cambio, creían que las nuevas transformaciones erosionarían la forma de producción campesina.

En el caso ecuatoriano, dos elementos claves debían articular los campesinos con el capitalismo: el acceso a la propiedad de la tierra y la venta de su fuerza de trabajo en el mercado, sobre todo urbano. El primer factor no tuvo éxito, debido a que el Ecuador no ha realizado una verdadera transformación de la estructura de tenencia de la tierra, mientras que el segundo factor fue muy efectivo. Con esto quiero señalar un primer punto de quiebre. Por un lado el acceso a la tierra ${ }^{4}$ es todavía un cabo suelto para el mundo campesino e indígena, a pesar de que las tierras comunales no fueron incorporadas al mercado de tierras tal como lo proponía la Ley de Desarrollo Agropecuario de 1994, y aunque no han sido vendidas, un gran porcentaje de ellas se encuentra repartido en usufructo individual. Esto ha implicado que el Estado haya mantenido las mejores tierras en pocas manos y se haya reservado la propiedad exclusiva de los recursos naturales no renovables que se encuentran en el subsuelo. En este contexto, el reclamo campesino de los sesenta sobre el acceso a la

${ }^{2}$ El Departamento de Antropología de la Pontificia Universidad Católica del Ecuador se creó en el año 1972.

${ }^{3}$ Según Lenin, la vía farmer implica la transformación de los campesinos en unidades familiares de producción, y la vía yunker la transformación de los campesinos a proletarios agrícolas.

${ }^{4}$ La concepción de "tierra" para los pueblos indígenas se va a convertir en "territorio" desde la vigencia del Convenio No. 169 de la OIT en 1989, y en el caso ecuatoriano en "circunscripciones territoriales indígenas" desde la Constitución de 1998. 
tierra mantiene plena vigencia en el siglo veintiuno y se ha ido transformando hasta convertirse formalmente en el derecho indígena a la delimitación y legalización de sus territorios, lo que constituye la base material y simbólica de otro derecho, el de la libre determinación, es decir, la tesis de la autonomía.

La venta de la fuerza de trabajo campesina ha experimentado otro derrotero y constituye un segundo punto de quiebre. Aunque al comienzo fue estigmatizada como una amenaza a la vigencia de la forma de producción campesina, con el tiempo su importancia ha cobrado relevancia. No hay que olvidar que los espacios de venta de fuerza de trabajo en un inicio tuvieron lugar en el ámbito rural agroindustrial, después pasaron al sector de servicios y comercio urbanos, para terminar en los mercados internacionales como resultado del proceso de inmigración masiva iniciado en los años ochenta hacia los Estados Unidos y luego a España e Italia, a partir del año 2000.

Así, Martínez y De Grammont recogen con acierto el trabajo pionero de Klein (1992), quien muestra que en América Latina el empleo rural no agrícola creció a mayor ritmo que el empleo agrícola durante la década de 1980 (Martínez y De Grammont, 2009: 11). Posteriormente, el trabajo de Reardon, Berdegué y Escobar (2001) demuestra que para la última década del siglo pasado, el 40 por ciento de los ingresos de la población rural latinoamericana provenía de actividades no agrícolas, y finalmente Kobrich y Dirven (2007), indican que a principios del siglo veintiuno, el 40 por ciento de la población ocupada lo hace en actividades no agrícolas. A este nuevo proceso, los autores mencionados anteriormente lo llaman "la pluriactividad" y constituye una estrategia central de las familias rurales (incluidas las indígenas), en el nuevo siglo.

La llamada "pluriactividad" plantea nuevos retos hacia el futuro ya que se trata de una estrategia de supervivencia para luchar contra el empobrecimiento de las familias campesinas, por la caída de los precios agropecuarios. Pero también puede entenderse como una estrategia de acumulación diversificada propia del desarrollo rural bajo el impulso de la globalización, lo que nos lleva inevitablemente a la necesidad de aplicar un enfoque multidisciplinar para entender la complejidad de la realidad rural ecuatoriana, caracterizada por un gran dinamismo.

Retomando el tema de la reforma agraria en Ecuador, es necesario incorporar otro elemento que incidió directamente en la vida de los pueblos indígenas y rurales ubicados en la Amazonía, como era el proceso paralelo de colonización en supuestas "tierras baldías". Me refiero al mecanismo que sirvió para adjudicar tierras de la Amazonía a los campesinos provenientes especialmente de la Sierra, pero sobre todo fue la manera usada por el Estado ecuatoriano y el capitalismo para incorporar a su funcionamiento a los pueblos indígenas de la Amazonía, que nada tenían de campesinos pero que formaban parte del mundo rural.

Este tema fue también de especial preocupación para la naciente antropología ecuatoriana. Autores como Bustamante (1988), por ejemplo, muestran que la mencionada adjudicación de tierras baldías afectó directamente a los territorios de las nueve nacionalidades indígenas que viven en la Amazonía, alterando dramáticamente su forma de vida. Este hecho dio inicio a un proceso moderno de etnogénesis 
de parte de las diversas organizaciones indígenas que a la par de reivindicar el territorio, también van a afirmar y recuperar sus lenguas, costumbres, cosmovisiones y valores 5 . En este tema es importante el aporte de Descola (1989) al analizar el caso de la nacionalidad Achuar.

Señalo este hecho como un tercer punto de quiebre porque desde inicios de la década de los setenta hasta la fecha, la región amazónica se va a convertir en la generadora de la mayor cantidad de divisas para el país con el inicio de la explotación petrolera, maderera y de monocultivos agroindustriales (palma africana). Simultáneamente estaba teniendo lugar el proceso de articulación de los pueblos indígenas con las empresa transnacionales ${ }^{6}$, quienes con la complicidad del Estado, han protagonizado una disputa totalmente desigual con los pueblos y nacionalidades indígenas del Ecuador que compromete seriamente su supervivencia y futuro.

En este punto quiero plantear un tema de debate resultado del proceso de etnogénesis que viven los pueblos indígenas amazónicos. Se trata del proceso de deterioro ambiental provocado por la explotación indiscriminada de los recursos naturales en contraste con el uso sostenible que dichos pueblos mantienen todavía en el centro sur de la Amazonia ecuatoriana. Este hecho, declarado mundialmente en muchas convenciones internacionales como una amenaza a la sostenibilidad del planeta, aparece ahora como una opción casi única de respuesta. La pregunta es ¿cómo conciliar el reconocimiento más avanzado de los derechos indígenas a nivel internacio$\mathrm{nal}^{7}$ con el máximo momento de expansión del capital globalizador ${ }^{8}$ ?

Otro de los debates de los estudios de la sociología de los años sesenta era el énfasis mostrado hacia la importancia de la categoría "clase social", en detrimento del factor étnico cuando se trataba de explicar el mundo agrario. Esta afirmación común, como señala Martínez (2007), es cuestionada por la producción antropológica de la época. Autores como Burgos (1970), y Villavicencio (1973), van a aplicar la propuesta de Aguirre Beltrán (1967), para el caso de México sobre regiones de refugio, colonialismo interno y proceso dominical. En el caso de Riobamba y Otavalo, las relaciones inter-étnicas que encubrían al mundo indígena y al mundo mestizo esta-

${ }^{5}$ El caso más pionero es la actual Federación Interprovincial de Centros Shuar (FICSH) que surge en 1964 con el apoyo de la misión salesiana para luego tener vida propia y convertirse en uno de los pilares para la creación de la futura Confederación de Nacionalidades Indígenas del Ecuador (CONAIE) a fines de los años ochenta.

${ }^{6}$ Desde 1972 cerca de 70 empresas petroleras de explotación y subsidiarias han invertido sus recursos en la Amazonia ecuatoriana y los pueblos indígenas han tenido necesariamente que "negociar" con ellas.

${ }^{7}$ Me refiero a la Declaración de Naciones Unidas sobre los Derechos de los Pueblos Indígenas aprobada en septiembre del año 2007.

${ }^{8}$ Aprovecho este tema para mencionar una de las propuestas del gobierno de Rafael Correa en el año 2007. Se trata del "Proyecto Yasuní": dejar el crudo en el subsuelo, que pretendía dejar de explotar 920.000 millones de barriles del bloque ITT con el apoyo de la cooperación internacional a través de la venta de carbono $(\mathrm{CO} 2)$. Esta iniciativa tras seis años de vigencia fue suspendida definitivamente por el gobierno de Correa el 15 de agosto de 2013, luego de lo cual se inició la explotación petrolera en esta reserva de biodiversidad. 
ban acompañadas de las relaciones de clase para mantener la explotación económica de un grupo sobre otro.

En el estudio de Villavicencio, la autora deja entrever un elemento que con el tiempo va a consolidarse, la aparición en los años setenta de un grupo de artesanos y comerciantes indígenas otavaleños que utilizando elementos propios de su identidad (los textiles, la música, la lengua y el parentesco) y el trabajo de jornaleros indígenas pobres, inician un proceso de acumulación económica que les convertirá en la actual burguesía indígena otavaleña. Además de lograr poder económico, esta burguesía indígena también se ha apropiado del poder político local. Este hecho, que para el director del Instituto Indigenista Interamericano de la época, el ecuatoriano Rubio Orbe, le parecía una conclusión peligrosa de Villavicencio, ha cobrado total vigencia.

Las relaciones de clase y étnicas cobran con el tiempo la doble función que les caracteriza: sirven para dominar y ser dominados, pero no solo entre mestizos e indios sino también entre indios. Estudios posteriores sobre este tema hechos por colegas ecuatorianos, incluidas dos intelectuales indígenas, (Torres, 2005; Maldonado, 2004 y Sarabino, 2007) y ecuatorianistas (Kyle, 2000; Meisch, 2002; ColloredoMansfeld, 1999), muestran con detenimiento esta afirmación.

Aquí surge un cuarto punto de quiebre; me refiero a la ambivalencia en América Latina de formas de resistencia al avance del capitalismo neoliberal protagonizadas por sectores de los pueblos indígenas. Éstas adoptan diversas formas de colaboración e incorporación activa al modelo neoliberal de mercado y se han servido de él para explotar a sus pares. Ejemplos parecidos al ecuatoriano encontramos entre los comerciantes indígenas de Bolivia, Perú, Guatemala y México y nos permiten plantear la siguiente pregunta: ¿de qué manera la denominada renta de la identidad es utilizada para la acumulación de poder económico y político por parte de la burguesía indígena?

Otro elemento que la antropología y la sociología ecuatoriana de los años sesenta incorporó a su agenda de investigación, fue el tema de la comunidad andina. Para su comprensión es necesario precisar algunos antecedentes. El marxismo latinoamericano antes de la revolución rusa de 1917, influenciado por la ideología del socialismo utópico y del anarco sindicalismo, consideraba al indio como un componente indiferenciado de un "pueblo" o de un "proletariado" igualmente oprimido, sin reconocer ningún carácter específico a su situación. El escritor y político peruano, Manuel González Prada fue quien planteó en su obra Nuestros Indios (1904), que el problema del indio no era un problema racial, ni tampoco era un problema cultural que encuentre su posible solución en la educación. Para él, se trataba de un problema esencialmente económico y social. Esta posición va a ser retomada por otros dos autores peruanos muy influyentes en su época: José Carlos Mariátegui e Hildebrando Castro Pozo, quienes en sus obras los Siete ensayos de interpretación de la realidad peruana (1928), y Nuestra comunidad indigena (1924), van a incorporar dos temas de trascendencia en la antropología latinoamericana, el de la sobrevivencia de la comunidad indígena o ayllu y el concepto de nacionalidad indígena.

Desde México, por su parte, Vicente Lombardo Toledano, después de su visita a la Unión Soviética, regresó impresionado por la manera como se resolvió allá el 
problema de las nacionalidades, e igualmente las compara con las nacionalidades indígenas en su libro Un viaje al mundo del porvenir (1936), en el que plantea, que se deberían crear grandes centros industriales en las áreas indígenas para explotar los recursos locales, lo cual arrancaría a los indios del campo, los proletarizaría y despertaría su conciencia revolucionaria. En otras palabras, a pesar de las evidencias de la realidad mexicana, se niega a definir a México como Estado plurinacional.

Hacia finales de los años veinte, la Tercera Internacional trata de orientar a los partidos comunistas latinoamericanos en los temas indígenas. Así, en la Conferencia Comunista Latinoamericana celebrada en Buenos Aires en 1929, se examina si en la lucha antiimperialista se debe incluir a las nacionalidades indígenas y la manera de hacerlo. Los dirigentes del entonces Komintern, nacionalistas en alto grado, consideran que el socialismo es el medio más rápido y seguro de construir la nación, y por lo tanto, consideran que el reconocimiento de las nacionalidades indias, siguiendo la tesis planteadas por Radek y Rosa Luxemburgo, desembocaría en la creación de Estados burgueses que retrasarían la revolución.

Respecto al pensamiento indigenista sobre la comunidad, el iniciador de esta tendencia en el Ecuador fue Pío Jaramillo Alvarado, y su principal contribución fue su obra publicada en 1922: El Indio Ecuatoriano. Contribución al estudio de la sociología indo-americana. La posición de Jaramillo se basó en la necesidad de incorporar al indio a la nación sin considerar lo que significaba y valía su propia cultura, con la recomendación de la "vuelta a la comunidad" o ayllu como la mejor forma de encontrar su unidad social. Además de su influencia en los estudios sociales, la literatura y el arte, ejerció importantes cargos públicos (diputado, senador y ministro de gobierno), lo que le permitió proponer soluciones en la organización sindical y en la legislación social ecuatoriana. Durante le década de 1930 a 1940 participó activamente en la aprobación de la legislación social que caracterizó la década de los treinta, en especial, durante la Asamblea Constitucional de 1937-1938 y en la dictadura del general Alberto Enríquez Gallo (1938), de tendencia izquierdista. Me refiero principalmente a la ley y estatuto jurídico que legalizó las comunas campesinas e indígenas, la ley de matrimonio civil y los códigos de menores y de trabajo. Toda esta legislación reconocía por primera vez una serie de derechos de los sectores populares ecuatorianos, especialmente de obreros, campesinos e indígenas. Es importante observar la participación de intelectuales mexicanos en estos avances, específicamente Vicente Lombardo Toledano en el código del trabajo9 y Moisés Sáenz en el tema indígena ${ }^{10}$.

${ }^{9}$ Vicente Lombardo Toledano, discípulo de Antonio Caso aunque luego rompió con él, creó la Confederación de Trabajadores de México, CTM, en 1936, y en 1938 fue director de la Confederación de Trabajadores de América Latina, CTAL.

${ }^{10}$ Moisés Sáenz, discípulo de Gamio, fundó en 1932 la primera Estación Experimental de Incorporación del Indio en Carapán, entre los Tarascos de Michoacán, antecedente de los futuros Centros Coordinadores del Instituto Nacional Indigenista. Entre enero de 1934 y enero de 1935 fue embajador de México en Ecuador, lo que le sirvió para conocer el país y escribir el libro Sobre el indio ecuatoriano y su incorporación al medio nacional, publicado en México por la Secretaría de Educación Pública en 1943. 
En los años setenta, hay dos aportes importantes en este tema tanto en los Andes como en Mesoamérica, que van a ser impulsados en forma simultánea desde la academia norteamericana y latinoamericana. Me refiero a la propuesta de pensadores marxistas que recogen la discusión de la formación del Estado, y en especial de las sociedades hidráulicas, para el caso de los pueblos mayas, aztecas e incas. Se trata de los trabajos de Karl Wittfogel (1966), Stanley Diamond (1979), Pedro Carrasco (1986), Richard Schaedel (1978) y John Murra (1978), que poco después se vincularon con las etnografías de las sociedades campesinas e indígenas contemporáneas.

En realidad, tanto Palerm desde México a través primero del CISINAH (Centro de Investigaciones Superiores del Instituto Nacional de Antropología e Historia) y luego del CIESAS (Centro de Investigaciones y Estudios Superiores en Antropología Social) como Murra ${ }^{11}$ desde los Andes a través del Instituto de Estudios Andinos, se van a convertir en los impulsores de la investigación etnohistórica de ambas realidades, y la amistad que les unió, dio como resultado la formación de grupos de investigadores locales que realizaron importantes contribuciones a la antropología tanto de los Estados Unidos como de México.

Siguiendo el trabajo iniciado por estos autores, es necesario señalar que en el Ecuador se realizaron varias contribuciones importantes como es el caso de los antropólogos de la Universidad Católica del Ecuador (Iturralde, 1980; Farga y Almeida, 1981; Moreno, 1977); la generada a partir de los investigadores del Centro Andino de Acción Popular (CAAP), y los trabajos de Martínez (1987), Santana (1993), Salomon (1980) y Bretón (2001), que de alguna manera buscaban en la comunidad indígena una vía para preservar el avance del capitalismo, así como utilizar la etnohistoria junto con elaboraciones etnográficas contemporáneas. Toda esta reflexión me sirve para postular un quinto punto de quiebre en el desarrollo de este trabajo. Materialmente a partir de la década de los noventa la discusión sobre la comunidad andina se desvanece en el mundo académico ecuatoriano. Queda reducida a un telón de fondo que sirve para el debate de otros temas más importantes con fuerte contenido y potencial "étnico", tales como la identidad, la justicia, los derechos individuales y colectivos, la educación y la salud intercultural, el género, la migración nacional e internacional, las fiestas y celebraciones, los recursos naturales, los movimientos sociales y políticos y la participación electoral.

Las tensiones que plantea este aparente olvido colectivo resultan interesantes para nuestro análisis, ¿cuál es la relación entre la comunidad y su base territorial? ¿acaso ahora asistimos a la existencia de las comunidades desterritorializadas? ¿la población indígena urbana, ha recreado la comunidad en un nuevo espacio? o ¿mantiene los lazos esporádicos con la comunidad rural original? Algo similar se puede decir de los migrantes internacionales, ¿acaso la comunidad diaspórica se ha convertido en el equivalente a la comunidad de origen? ¿Qué define a la comunidad como eje organizativo de los diferentes modelos autonómicos indígenas?

${ }^{11}$ El primer contacto de Murra con los Andes fue en el año 1941, cuando llego al Ecuador para hacer una investigación arqueológica sobre la presencia de la cultura Chavin en los Andes del norte, como parte del proyecto de Donald Collier, profesor de la universidad de Chicago (Castro, Aldunate e Hidalgo, 2000). 
Aníbal Buitrón es considerado el primer antropólogo ecuatoriano de carrera. En 1945 se doctoró en la universidad de Chicago, e inició junto con su esposa Bárbara Salisbury también antropóloga, investigaciones sobre la zona de Otavalo. Su obra más representativa fue la que escribió con su colega John Collier Jr. en 1949, denominada El valle del amanecer, publicada por la Universidad de Chicago. Buitrón fue funcionario y consultor internacional de Naciones Unidas por muchos años e impulsor de la política de desarrollo de la comunidad, además de académico de algunas universidades norteamericanas. Menciono este autor porque junto con Burgos y Villavicencio, son los primeros autores en hacer crudas descripciones sobre la cuestión de la discriminación sufrida por los indígenas por parte de la población blanca y mestiza, en especial en los espacios públicos y en las instituciones públicas.

De forma simultánea, la publicación de las obras de estos antropólogos ecuatorianos coincide con la ratificación que hace el gobierno ecuatoriano de la Convención Internacional sobre todas las formas de discriminación racial (CERD) el 4 de enero de 1969, que luego fue complementada por la Convención Internacional contra el Racismo, la Discriminación, la Xenofobia y otras formas de intolerancia, el 8 de septiembre del 2001. La antropología y sociología ecuatorianas de fines del siglo pasado retomaron con fuerza este tema, en especial los trabajos de Almeida (1996), De la Torre (1996 y 2002), Cervone y Rivera (1999) y Rahier (2003). Estas contribuciones, junto a la Encuesta Nacional sobre Racismo y Discriminación Racial en el Ecuador realizada en el 2004 por la Secretaría Técnica del Frente Social —STFS - y el Instituto Nacional de Estadísticas y Censos (INEC), muestran que a pesar del reconocimiento social y político logrado por el movimiento indígena y por el naciente movimiento afroecuatoriano, la práctica del racismo sigue vigente en el país ${ }^{12}$. La encuesta se convirtió en la primera experiencia gubernamental de esta naturaleza en los países de América Latina y el Caribe, y respondió tanto a las demandas de las organizaciones de la sociedad civil afroecuatoriana, como al mandato del Plan de Acción de la Tercera Conferencia Mundial contra el Racismo y la Discriminación Racial, celebrada en Durban, Sudáfrica, en el 2001.

Aunque el racismo sea un fenómeno difícil de medir dado que es un componente total de la estructura social que se manifiesta en ideologías, sentimientos y prácticas de dominación, poder, opresión y exclusión política y socioeconómica por parte de actores sociales (INEC y Secretaria Técnica del Frente Social, 2004), la encuesta indagó datos que evidencian que esta práctica aun implica conflictos de identidad, impide la concreción de la interculturalidad como proyecto nacional y sobre todo, se convierte en un obstáculo para el disfrute de los derechos humanos por parte de sectores históricamente discriminados, como los indígenas y afrodescendientes.

Los datos son contradictorios (INEC y Secretaria Técnica del Frente Social, 2004) porque aunque el $62 \%$ de los ecuatorianos admite que en el país existe el racismo y

${ }^{12}$ La encuesta nacional (INEC y Secretaria Técnica del Frente Social) fue ejecutada en el mes de septiembre del 2004 en 22 provincias del Ecuador, con el $65.6 \%$ urbano y el $34.4 \%$ rural, alcanzando una muestra representativa de 37.519 personas y 8.687 hogares, de los cuales el $81 \%$ se auto identificó mestizo, $6.6 \%$ blanco, $8.3 \%$ indígena y el $4 \%$ afroecuatoriano. Esta encuesta es hasta la fecha la única realizada en el país sobre el tema del racismo. 
la discriminación, solo el 10\% se considera abiertamente racista, siendo los blancos los más racistas (14\%), mientras los menos racistas son los afroecuatorianos (5\%). De acuerdo a la condición socio racial, se confirma que los afroecuatorianos, quienes lograron la libertad tras varios siglos de esclavitud apenas hace 155 años, son las mayores víctimas del racismo (88\%), seguidos por los indígenas (71\%). Justamente estos grupos son a su vez los más pobres del Ecuador según el indicador de necesidades básicas insatisfechas ( $70.1 \%$ y $90.1 \%$, respectivamente), poseen la tasa de analfabetismo más altas del país (10,2\% y $28.1 \%)$ y obtienen menos ingresos que blancos y mestizos.

En cuanto a la discriminación racial, entendida como practicas institucionales de exclusión, restricción o preferencia por motivos raciales y étnicos (INEC y Secretaria Técnica del Frente Social, 2004), la encuesta reveló que los afroecuatorianos son el grupo que más ha experimentado este fenómeno en el último año anterior a la encuesta (2003) con el $44 \%$ del total, siendo la calle (63\%), el barrio (38\%), los autobuses (37\%) y las oficinas públicas (36\%), los lugares donde más se ha sufrido la discriminación racial.

Uno de las aspectos que más preocupa, según los datos de la encuesta, es el tema de la discriminación laboral, donde justamente son los afroecuatorianos (13\%) y los indígenas $(10 \%)$ quienes más se han sentido discriminados en sus lugares de trabajo, bien sea por su patrón, sus compañeros de oficina o todo el personal de trabajo. Este problema de discriminación laboral es mucho más grave si tenemos en cuenta que en el Ecuador la tasa más alta de desempleo la poseen los afroecuatorianos con el 7\%, mientras que la tasa promedio nacional es del 5\%, según la Encuesta Nacional de Empleo y Desempleo Urbano de diciembre de 2011 (ENEMDU). Esta alta tasa de desempleo que constantemente caracteriza a los afrodescendientes del país, está en estrecha relación con su baja profesionalización (su tasa de asistencia neta universitaria es de $9.2 \%$, respecto al $25.4 \%$ de los blancos y al $21.5 \%$ de los mestizos (INEC, 2010).

La discriminación laboral racial y el desempleo igualmente se relacionan con el trato según el color de la piel. Según la encuesta, en el Ecuador los blancos tienen mejor oportunidades y son los más aceptados en los espacios sociales. Los datos revelan que el $80 \%$ de los ecuatorianos están de acuerdo con la afirmación de que los blancos son los que mejor trato reciben, pese a que solo son el $10.5 \%$ de la población. Mientras los mestizos, que son más del 77,6\% de la población, son aceptados y reciben buen trato en un $6 \%$. Por su parte, los que menos trato reciben son los indígenas y los afros con el $1 \%$.

El 63\% de los encuestados afirman que en el Ecuador existe prejuicio racial hacia los afroecuatorianos. Pero, contradictoriamente, apenas el 9\% afirma ser responsable del hecho. Ante esta ambigüedad y como una medida más exacta del diagnóstico del problema, la Secretaría Técnica a partir de varios indicadores sobre el prejuicio racial (acuerdo sobre frases ofensivas, actitudes sociales, estereotipos) diseñó el Índice de Prejuicio Racial Indirecto, el cual fue calculado en un $76 \%$, es decir, que de cada 7 ecuatorianos, 5 poseen algún grado de prejuicio frente a los afroecuatorianos, siendo los blancos e indígenas los más prejuiciosos ( 82 y $80 \%$, respectivamente), 
mientras que regionalmente, es la Sierra la que demuestra un mayor porcentaje de prejuicio racial $(80 \%)$, seguida de la Costa $(72 \%)$.

De la misma manera se calculó el Índice de cultura sobre el racismo, a partir de la indagación de conocimientos generales sobre aspectos que tienen que ver con la discriminación, tales como instrumentos jurídicos, derechos colectivos, el Plan de Acción de Naciones Unidas contra el racismo (Durban, 2001), etc. De acuerdo a los cálculos, el Índice de conocimiento de cultura contra el racismo es del $47 \%$, siendo los afrodescendientes y los blancos los que mayor cultura poseen.

La encuesta determinó que en Ecuador la responsabilidad del Racismo recae principalmente en el Gobierno Nacional (55\%), y luego en la ciudadanía (37\%). Sin embargo el $78 \%$ piensa que el mismo gobierno no hace nada por combatir la discriminación racial, por tanto más del $82 \%$ está de acuerdo con que el Estado desarrolle alguna medida para combatir este fenómeno. El 67\% de los encuestados está de acuerdo con que el Estado implemente medidas de acción afirmativa para combatir de forma efectiva la discriminación racial, de los cuales el $67 \%$ sugiere que la enseñanza de la diversidad étnica y cultural sea la política prioritaria, en tanto solo el 13\% propone oficinas para atender casos de discriminación, el 12\% propone campañas masivas en los medios, y solo el $8 \%$ está de acuerdo con leyes que penalicen el racismo y sus actos.

Esta larga disquisición me permite formular un sexto punto de quiebre, con sus consiguientes tensiones. Después de que el Ecuador aprobara la Constitución en el año 2008, y se haya autodefinido como un Estado de derecho, plurinacional e intercultural ¿hasta qué punto esa calidad es transversal a la estructura de la sociedad ecuatoriana? ¿O es todavía un discurso? La discriminación racial y la exclusión étnica cultural hacia los indígenas y los afrodescendientes es todavía una práctica cotidiana de la población blanca y mestiza, tanto en el sector rural como en el sector urbano. El gobierno ecuatoriano ha llevado a cabo una política pública en proceso de formulación y ejecución sobre interculturalidad e inclusión, que incluye un plan plurinacional contra la discriminación racial y la exclusión étnica cultural iniciado en el año 2009. Se trata tal vez de un intento de institucionalización de la diferencia en el marco de la reforma del Estado pero sin cuestionar la estructura económica y política de dominación. Este plan que ha estado vigente hasta el año 2012, no tiene al momento ninguna otra política pública que lo reemplace.

Un tema que la antropología ecuatoriana le ha dedicado tiempo y elaboración es el del género y etnicidad. Sin embargo, su vigencia no surge del mundo académico en una primera instancia, sino de la cooperación y legislación internacional y del interés del movimiento de mujeres de carácter más urbano y con militantes de la clase media. En un inicio se plantearon dos temas prioritarios: el reconocimiento de la desigualdad de género y la exigibilidad de los derechos de las mujeres. Van a ser algunas investigadoras ecuatorianistas las que primero investiguen a las mujeres rurales e indígenas (Stolen, 1987; Weismantel, 1988; Crain, 1991, 1996).

Los primeros trabajos se van a centrar en el papel de las mujeres indígenas en la economía campesina (Poeschel, 1986; Martínez, 1988), fue el trabajo pionero de Stolen (1987) sobre las mujeres rurales mestizas y el que va a plantear la situación 
de violencia y opresión que enfrentan estas mujeres. Esto ha permitido abrir un interesante debate que se ha mantenido hasta la actualidad: las tensiones que han surgido entre las reivindicaciones de las mujeres indígenas y muchas de sus costumbres culturales (la famosa complementariedad de género por ejemplo), que justifican su situación de exclusión.

Con frecuencia, la violencia y la opresión contra las mujeres indígenas se han interpretado como una influencia occidental o capitalista que ha enturbiado aquellas relaciones tradicionales descritas como armoniosas. Esta idea es cuestionada por Blanca Muratorio (2001), que usando fuentes orales y de archivo ha documentado que la violencia de género entre los napo-runas kichwas tiene raíces tanto internas a su propia tradición, como relacionadas con el proceso de colonización.

La presencia de las organizaciones de mujeres indígenas tanto de la Sierra como de la Amazonía ha marcado un interesante debate tanto al interior del movimiento indígena como en el movimiento de mujeres. En este sentido, quiero hacer referencia a tres hechos significativos que merecen ser descritos y analizados. El primero tiene que ver con la participación de estas organizaciones en el desarrollo y aprobación de la nueva Constitución ecuatoriana en el año 2008, concretamente con respecto al artículo 171, que norma la vigencia de la justicia indígena. Ésta se ha introducido en el texto constitucional señalándose que la aplicación de la justicia no sería posible sin "la garantía de participación y decisión de las mujeres", tras un debate con los asambleístas del movimiento Pachakutik que eran todos hombres y que se oponían a introducir esta parte del texto.

El segundo hecho se refiere a la declaración del Encuentro Internacional "Mujeres Indígenas y Justicia Ancestral", llevado a cabo en Quito del 20 al 24 de octubre de 2008, con la presencia de delegadas indígenas de nueve países latinoamericanos. En este Encuentro Internacional aunque se reconoció la existencia del sistema de justicia ancestral, se señaló que no se estaba atendiendo de manera satisfactoria y oportuna la resolución de una serie de conflictos que involucran a las mujeres, tales como la violencia en sus múltiples expresiones, adulterio, pago de alimentos, conflictos de herencia, acceso a la tierra e impedimento de su participación en las organizaciones representativas y en el nombramiento en calidad de autoridades.

El último hecho es continuación del anterior y tuvo lugar en febrero del año 2009 durante un encuentro internacional donde las organizaciones de mujeres indígenas ecuatorianas plantearon a las autoridades estatale ${ }^{13} \mathrm{y}$ a la cooperación internacional involucradas en la reforma de la normativa que se desprende de la aplicación de la nueva Constitución, su participación activa y militante con propuestas específicas. Luego del 2009 el diálogo entre el movimiento indígena (incluidas las mujeres) y el gobierno de Correa se interrumpió sin que a la fecha se haya restituido.

Para finalizar, planteo un séptimo punto de quiebre entre los estudios agrarios y étnicos. Nuevamente en este tema confluyen tres tensiones o campos de fuerza,

${ }^{13}$ En especial el Ministerio de Justicia y Derechos Humanos, La Fiscalía General, los Consejos de la Igualdad Étnicos y de la Mujer, el Plan Nacional contra la Violencia y el sistema de Naciones Unidas. 
tal y como señala Diego Iturralde en las discusiones realizadas por los 35 años del CIESAS entre sus investigadores. Dichas tensiones provienen de tres tipos de actores: las influencias de los organismos internacionales, las resultantes de la reforma del Estado y las provenientes de los movimientos sociales, en este caso el movimiento étnico y el movimiento feminista.

En este contexto, varias problemáticas se hacen ostensibles: una primera, sobre cómo conciliar las políticas de reconocimiento de los pueblos indígenas y afrodescendientes con las políticas de redistribución que son de aplicación general a todos los ciudadanos y ciudadanas de un mismo país, cuando tanto una como otra forman parte de lo que Boaventura de Sousa Santos (2003) llama "el equilibrio entre la igualdad y la diferencia". Las reivindicaciones de las mujeres en su conjunto, como las de las mujeres indígenas y afrodescendientes en específico, necesitan ser visibilizadas y ser tenidas en cuenta por las políticas públicas.

Otra problemática resulta de la confrontación que las mujeres indígenas han hecho de muchas de sus propias tradiciones y costumbres, sin que por esto signifique cambios y pérdida de su identidad colectiva. El caso de la violencia doméstica de los hombres contra las mujeres, niños y niñas, muestra que ciertas prácticas de exclusión mantenidas a través del tiempo han servido a los intereses patriarcales de los grupos de poder indígenas, que en su gran mayoría están constituidos por hombres. Este ejercicio ha respondido al intento de algunos investigadores indígenas y no indígenas por idealizar la integridad cultural de estos pueblos, cayendo en posiciones esencialistas desde el punto de vista étnico.

Por último, el ejemplo mencionado de las acciones de las mujeres indígenas ecuatorianas demuestra que sí es posible conciliar al interior del mundo indígena las demandas de género con las demandas colectivas de sus pueblos, lo que ha implicado una negociación tanto del discurso como de las prácticas respecto a la cultura, los derechos y las relaciones de poder entre hombres y mujeres.

A manera de cierre, y luego de la revisión de los siete puntos de quiebre entre los estudios agrarios y los estudios étnicos en Ecuador, podemos señalar que a pesar de los avances logrados en el país luego de las Constituciones de 1998 y 2008, en el tema de derechos colectivos para indígenas y afrodescendientes, su aplicación a la fecha es limitada y el paso de la norma a su cumplimiento y garantía es tarea pendiente del conjunto de la sociedad ecuatoriana, en especial la definición del uso, manejo y control de los recursos estratégicos en el sector agrario como son la tierra, el agua, los bosques y los recursos del subsuelo.

\section{Referencias bibliográfícas}

\section{AGUIRRE BELTRÁN, Gonzalo}

1967 Regiones de Refugio. El desarrollo de la comunidad y el proceso dominical en mestizo América. México: Instituto Indigenista Interamericano.

ALMEIDA, José

1996 "Fundamentos del racismo ecuatoriano," Ecuador Debate No. 38, Quito: CAAP, pp. 55-71. 
BRETON, Víctor

2001 Cooperación al desarrollo y demandas étnicas en los Andes ecuatorianos. Ensayo sobre indigenismo, desarrollo rural y neo indigenismo. Quito: FLACSO Ecuador, Universidad de Lleida y GIEDEM.

BURGOS, Hugo

1997 [1970] Relaciones interétnicas en Riobamba. Quito: Corporación Editora Nacional.

BUSTAMANTE, Teodoro

1988 La larga lucha del kakaram contra el sucre, Quito:Abya Yala.

CARRASCO, Pedro

1986 La sociedad indígena en el centro y occidente de México. México: El Colegio de México.

CASTRO, Victoria; ALDUNATE, Carlos; HIDALGO, Jorge (Eds.)

2000 Nispa Ninchis. Conversaciones con John Murra. Lima: Instituto de Estudios Peruanos e Instituto de Estudios Andinos.

CERVONE, Emma; RIVERA, Freddy

1999 Ecuador racista: imágenes e identidades, Quito: FLACSO.

CHAYANOV, Alexander

1974 La organización de la unidad económica campesina. Buenos Aires: Ediciones Nueva Visión.

COLLOREDO-MANSFELD, Rudy

1999 Native Leisure Class. Consumption and cultural creativity in the Andes. Chicago: The University of Chicago Press.

CRAIN, Mary

1991 "Poetics and Politics in the Ecuadorian Andes: Women's Narratives of Death and Devil Possession," American Ethnologist vol. 18, num. 1.

1996 "The Gendering of Ethnicity in the Ecuadorian Andes," en A. STOLEN y M. MELHUS (Eds.), Machos, Mistresses and Madonnas, New York: Verso.

DE LA TORRE, Carlos

1996 El racismo en el Ecuador. Experiencias de los indios de clase media. Quito: CAAP.

2002 Afroquiteños: Ciudadanía y racismo. Quito: CAAP.

DESCOLA, Philippe

1989 La Selva Culta. Quito: Abya-Yala.

DIAMOND, Stanley

1979 Toward a Marxist Anthropology: Problems and Perspectives. Great Britain: Mouton Publishers.

FARGA, Cristina; ALMEIDA, José

1981 Campesinos y comunidades. Otavalo: Instituto Otavaleño de Antropología. 
INSTITUTO NACIONAL DE ESTADÍSTICAS Y CENSOS (INEC) y SECRETARIA TÉCNICA DEL FRENTE SOCIAL

2004 Primera Encuesta Nacional sobre Racismo y Discriminación Racial en el Ecuador. Quito: Ediciones INEC.

\section{INSTITUTO NACIONAL DE ESTADÍSTICAS Y CENSOS (INEC)}

2010 VII Censo de Población. Quito: Ediciones INEC.

2011 Encuesta Nacional de Empleo y Desempleo Urbano (ENEMDU). Quito: Ediciones INEC.

ITURRALDE, Diego

1980 Guamote: Campesinos y comunas. Otavalo: Instituto Otavaleño de Antropología.

KYLE, David

2000 Transnational peasants: migration, networks and ethnicity in Andean Ecuador. Baltimore: The Johns Hopkins University Press.

KLEIN, Emilio

1992 "El empleo rural no agrícola en América Latina". Documentos de Trabajo, No. 364, agosto, Santiago de Chile, PREALC, Programa Mundial del Empleo, OIT.

KOBRICH, Claus; DIRVEN, Martine

2007 Características del empleo rural no agrícola en América Latina con énfasis en los servicios. Santiago de Chile: CEPAL.

MALDONADO, Gina

2004 Comerciantes y viajeros, de la imagen etnoarqueológica de "lo indígena” al imaginario del kichwa Otavalo "universal”. Quito: FLACSO-Abaya-Yala.

MARTÍNEZ, Alexandra

1998 "La producción de esteras en Yahuarcocha y la construcción del significado de ser mujer y ser hombre,” en LANDÁZURI, Cristóbal. (Coord.), Memorias del primer congreso ecuatoriano de antropología, Quito: PUCE-Marka.

MARTÍNEZ, Carmen

2007 "Antropología indigenista en el Ecuador desde la década de 1970: compromisos políticos, religiosos y tecnocráticos", en Revista Colombiana de Antropología, volumen 43, enero-diciembre de 2007, 335-366.

MARTÍNEZ, Luciano y Henry DE GRAMMONT, (Comps.)

2009 La pluriactividad en el campo latinoamericano. Quito: FLACSO.

MARTÍNEZ, Luciano

1987 Economía política de las comunidades indígenas. Quito: Editorial El Conejo.

MEISCH, Lynn

2002 Andean entrepreneurs, otavalo merchants and musicians in the global arena. Austin: University Texas Press.

MINTZ, Sidney

1973 “A Note on the Definition of Peasantries". Journal of Peasant Studies 1(1): 91-106. 
MORENO, Segundo

1977 Sublevaciones indígenas en la Audiencia de Quito desde comienzos del siglo XVIII hasta finales de la colonia. Quito: Centro de Publicaciones, PUCE.

MURRA, John V.

1978 La organización económica del Estado Inca. México: Siglo XXI Editores.

MURATORIO, Blanca

2001 "History and Cultural Memory of Violence against Indigenous Women in the Ecuadorian Upper Amazon," manuscrito.

POESCHEL, Ursula

1986 La mujer salasaca. Quito: Abya Yala.

RAHIER, Jean

1998 "Blackness, the Racial Spacial Order, Migrations and Miss Ecuador 1995-1996", American Anthropologist vol. 100: 2, 421-430.

REARDON, Thomas; BERDEGUÉ, Julio; ESCOBAR, Germán

2001 "Rural Nonfarm Employment and Incomes in Latin American: Overview and Policy Implications", Word Development, 29 (3): 395-409.

SALOMON, Frank

1980. Los señores étnicos de Quito en la época de los incas. Otavalo: Instituto Otavaleño de Antropología.

SANTANA, Roberto

1993 “Globalización de la economía y campesinado serrano: un análisis en tres dimensiones”, Ecuador Debate No. 29. CAAP, Quito.

SANTOS, Boaventura de Sousa

2003 La caída del angelus novus: ensayos para una nueva teoría social y una nueva práctica política, Bogotá: ILSA.

SARABINO, Zoila

2007 El proceso de construcción de las elites indígenas en la ciudad de Otavalo. Tesis de Maestría, FLACSO.

SCHAEDEL, Richard

1978 "Early state of the Incas", CLAESSEN, Henry J.M; SKALNIK, Peter (Eds.). Early State. Great Britain: Mouton Publishers, The Hague, The Netherlands.

STOLEN, Anne Kristine

1987 A media voz: relaciones de género en la sierra ecuatoriana, Quito: CEPLAES.

TORRES, Alicia

2005 "De Punyaro a Sabadell...la emigración de los kichwa Otavalo a Cataluña" en G. HERRERA, C. CARRILLO y A. TORRES (Eds.), La migración ecuatoriana: transnacionalismo, redes e identidades. Quito: FLACSO, 433-448. 
VILLAVICENCIO, Gladis

1973 Relaciones interétnicas en Otavalo. ¿Una nacionalidad india en formación?, México: Instituto Indigenista Interamericano, México.

WEISMANTEL, Mary

1988 Food, Gender, and Poverty in the Ecuadorian Andes, Philadelphia: University of Pennsylvania Press.

WITTFOGEL, Karl August

1966 Despotismo oriental: estudio comparativo del poder totalitario. Madrid: Guadarrama.

WOLF, Eric

1977 Una tipología del campesinado latinoamericano. Buenos Aires: Ediciones Nueva Visión. 